\title{
PREFACE: THEORETICAL AND MATHEMATICAL MODELING OF FLOW AND TRANSPORT IN POROUS MEDIA
}

Theoretical and mathematical modeling approaches are of great importance in studying the flow and transport properties of porous media for all scientific, engineering, and environmental applications. In recent decades, analysis of flow and transport in porous media in many fields, such as soil science, polymer composite, chemical engineering, materials science, groundwater, and petroleum engineering, has received substantial attention, as seen in Fig. 1.

This special issue emphasizes fundamental scientific innovations related to the flow and transport properties of porous media that will increase our basic understanding of theoretical and mathematical modeling of transport phenomena in porous media. The works presented in this issue on the theoretical and mathematical modeling and approaches can be helpful for improving our understanding of flow and transport in porous media.

Papers published in this issue cover a wide range of applications using different theoretical and mathematical approaches and analysis of flow and transport phenomena, and they can be divided into two groups.

The first group of papers consists of five works that focus on fluid flow in porous media. Within the first group, the work "A Fast Calculation Method for Estimating the Representative Elementary Volume of a ThreeDimensional Fracture Network" by Huang et al. proposes a new approach to estimate the representative elementary volume of the three-dimensional discrete fracture network model in which fractures are modeled as circular disks of arbitrary size, orientation, and location. Their developed approach has the potential application to quantify the fluid flow process through complex porous media by using the geometric parameters of a fracture network. Based on the influences of the real gas PVT variation on well deliverability under formation conditions and the non-Darcy effects, the work "Investigation of the Productivity Prediction Model of the Acid Fracturing Well Considering the Real Gas PVT" by Xiong et al. proposes a productivity prediction model of the finite conductivity vertical acid fracturing well in a low-permeability reservoir by considering the slippage effect, threshold pressure gradient, and stress sensitivity. The work "Soret and Dufour Effects on Radiation Absorption Fluid in the Presence of
Exponentially Varying Temperature and Concentration in a Conducting Field" by Harinath Reddy et al. presents a numerical analysis for the case of Soret and Dufour effects on radiation absorption of a fluid past a linearly accelerated vertical porous plate in the presence of exponentially varying temperature and concentration in a conducting field. The work "Effect of Unsaturated Seepage on Slope Stability under Rainfall" by Cao et al. investigates the process of one-dimensional unsaturated soil seepage by means of fractal technology, and a new formulation to define the critical depth for slope failure under rainfall is presented. The final paper within the first group is entitled "A Full Approximate Analytic Solution to the Problem of Spontaneous Imbibition in Porous Media." In this work the author, Mirzaei-Paiaman, analyzes the problem of spontaneous imbibition in porous media with the gravity effect included, leading to a full approximate analytical solution using the Lambert $\mathrm{W}$ function.

The second group of papers includes five works that focus on heat conduction in porous media. The article "Numerical Analysis of Heat Conduction Treated with Highly Conductive Copper Oxide Nanoparticles in Porous Media" by Rokhforouz et al. presents a mathematical investigation of the effect of highly conductive copper oxide nanoparticles on the effective thermal conductivity of rock samples. Their results show that conductive heat transfer through porous media is affected by copper oxide nanofluid, porosity, and nanoparticle concentration, and confirms that the copper oxide nanoparticles can be used for the improvement of heat transfer in rock-fluid samples. The article "Free Convective Heat and Mass Transfer Flow of Heat-Generating Nanofluid Past a Vertical Moving Porous Plate in a Conducting Field" by Chandra Reddy et al. addresses the free convective heat and mass transfer characteristics of a heat-generating magneto-nanofluid flow through a vertical moving porous plate within a conducting field, in which the water-based nanofluid with the composition of copper nanoparticles is considered. The article "Electrothermo Convection of Rotating Nanofluid in Brinkman Porous Medium" by Chand and Rana presents an analytical study of the thermal convection in a horizontal layer of rotating nanofluid with 
Cai et al.
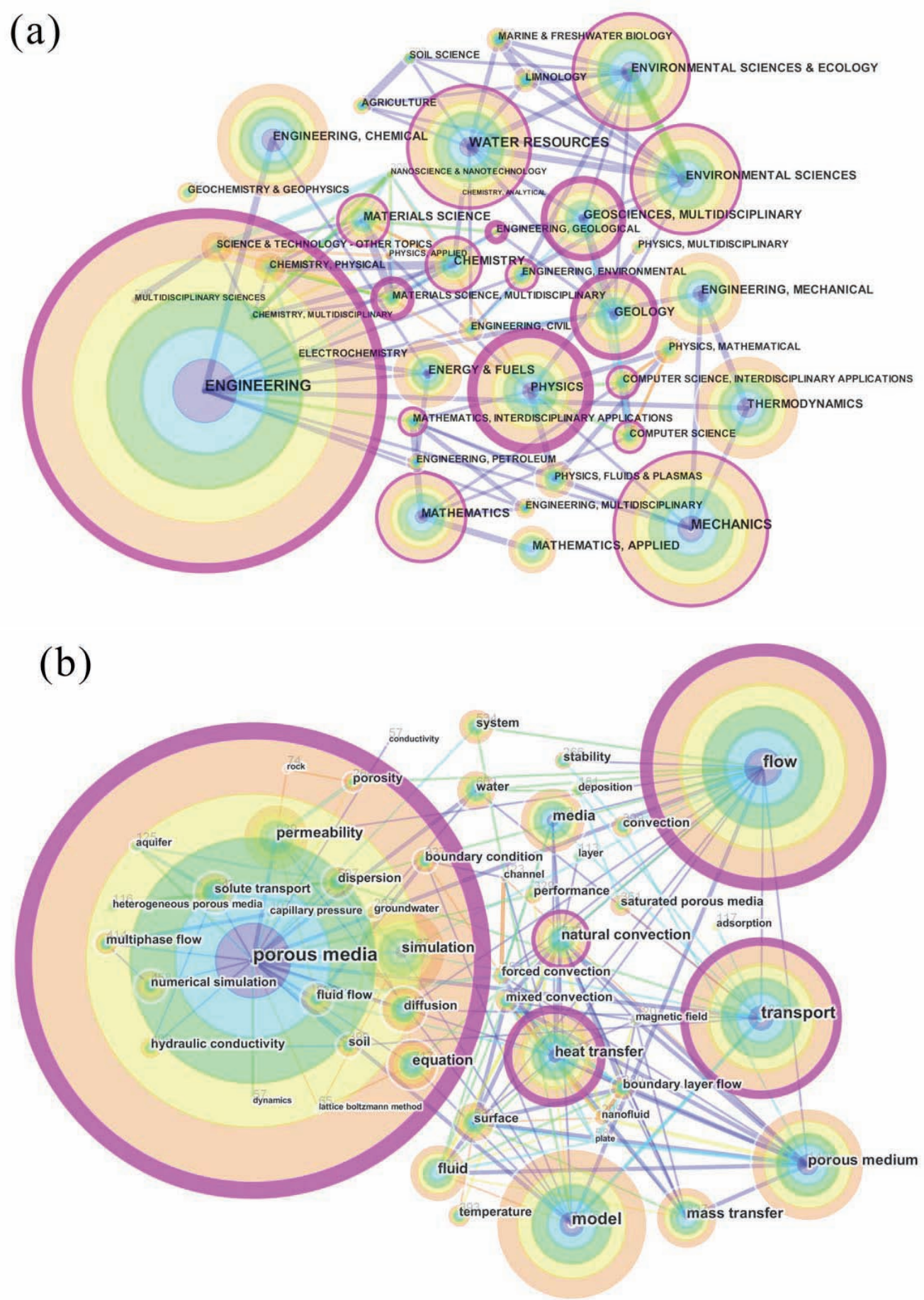

FIG. 1: Over 12,000 papers were published within the 2011-15 time frame related directly or indirectly to various aspects of flow and/or transport in porous media: (a) category and (b) keywords. [This data is adapted from the Web of Science Core Collection (SCI-Expanded)]. 
vertical $\mathrm{AC}$ electric field in a porous medium, including the effects of Brownian diffusion, thermophoresis, and electrophoresis. The article "Influence of Thermal Radiation and Thermophoresis on Viscoelastic Fluid Flow over a Vertical Cone" by Rushi Kumar et al. looks at the unsteady, free convective heat and mass transfer problem of a Walters-B viscoelastic non-Darcy flow over a vertical cone while incorporating the influence of thermal radiation, thermophoresis, and higher order chemical reactions. The article "Effects of Joule Heating on MHD Free Convective Flow Along a Moving Vertical Plate in Porous Medium," by Chandrasekhar Reddy et al., presents an investigation of the effects of Joule heating on an unsteady MHD free convective heat and mass transfer flow past a moving vertical plate in a porous medium in the presence of a chemical reaction.

\section{Guest Editors:}

\section{Jianchao Cai}

Hubei Subsurface Multi-Scale Imaging Key Laboratory, Institute of Geophysics and Geomatics, China University of Geosciences,

Wuhan, P. R. China

\section{Kambiz Vafai}

Department of Mechanical Engineering, University of California, Riverside, Riverside, CA, USA
In conclusion, the use of theoretical and mathematical methods to describe the flow and transport in porous media is an extremely active field of research in many different disciplines. The presented papers summarize some of the existing theoretical and mathematical approaches and their applications to flow and transport in porous materials.

The guest editors would like to acknowledge all of the authors of the special issue for their inspiring contributions and the anonymous referees for their diligent work, which ensures the high quality of this issue. Guest editor Jianchao Cai would like to thank Prof. Kambiz Vafai and Prof. Moran Wang for their great help and cooperation while editing this issue, and the National Natural Science Foundation of China (No. 41572116) for supporting his series of research on flow and transport in porous media.

\section{Abouzar Mirzaei-Paiaman}

Department of Petroleum Engineering, National Iranian South Oil Company,

Ahvaz, Iran

Fuyong Wang

Research Institute of Enhanced Oil Recovery, China University of Petroleum, Beijing, P. R. China 